\title{
Amos, Karin
}

\section{Aspekte der angloamerikanischen pädagogischen Differenzdebatte: Überlegungen zur Kontextualisierung}

formal überarbeitete Version der Originalveröffentlichung in:

formally revised edition of the original source in:

Lutz, Helma [Hrsg.]; Wenning, Norbert [Hrsg.]: Unterschiedlich verschieden. Differenz in der Erziehungswissenschaft. Opladen : Leske + Budrich 2001, S. 71-92

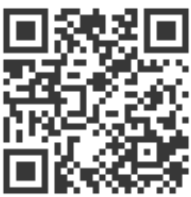

Bitte verwenden Sie in der Quellenangabe folgende URN oder DOI /

Please use the following URN or DOI for reference:

urn:nbn:de:0111-opus-26091

10.25656/01:2609

https://nbn-resolving.org/urn:nbn:de:0111-opus-26091

https://doi.org/10.25656/01:2609

\section{Nutzungsbedingungen}

Gewährt wird ein nicht exklusives, nicht übertragbares, persönliches und beschränktes Recht auf Nutzung dieses Dokuments. Dieses Dokument ist ausschließlich für den persönlichen, nicht-kommerziellen Gebrauch bestimmt. Die Nutzung stellt keine Übertragung des Eigentumsrechts an diesem Dokument dar und gilt vorbehaltlich der folgenden Einschränkungen Auf sämtlichen Kopien dieses Dokuments müssen alle Urheberrechtshinweise und sonstigen Hinweise auf gesetzlichen Schutz beibehalten werden. Sie dürfen dieses Dokument nicht in irgendeiner Weise abändern, noch dürfen Sie dieses Dokument für öffentliche oder kommerzielle Zwecke vervielfältigen, öffentlich ausstellen, aufführen, vertreiben oder anderweitig nutzen.

Mit der Verwendung dieses Dokuments erkennen Sie die Nutzungsbedingungen an.

\section{Terms of use}

We grant a non-exclusive, non-transferable, individual and limited right to using this document

This document is solely intended for your personal, non-commercial use. Use of this document does not include any transfer of property rights and it is conditional to the following limitations: All of the copies of this documents must retain all copyright information and other information regarding legal protection. You are not allowed to alter this document in any way, to copy it for public or commercial purposes, to exhibit the document in public, to perform, distribute or otherwise use the document in public.

By using this particular document, you accept the above-stated conditions of use.

\section{Kontakt / Contact:}

\section{peDOCS}

DIPF | Leibniz-Institut für Bildungsforschung und Bildungsinformation Informationszentrum (IZ) Bildung

E-Mail:pedocs@dipf.de

Internet: www.pedocs.de

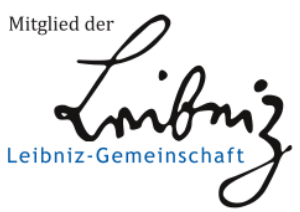


Helma Lutz/Norbert Wenning (Hrsg.)

\section{Unterschiedlich verschieden \\ Differenz in der Erziehungswissenschaft}

Leske + Budrich, Opladen 2001 
Die erste Auflage dieses Band erschien 2001 im Verlag Leske + Budrich, Opladen (jetzt VS-Verlag, Wiesbaden) und hatte folgende ISBN:

ISBN 3-8100-2854-1

Diese Version des Textes wird von der Herausgeberin und dem Herausgeber verantwortet. Sie macht den Gesamttext mit allen Beiträgen des genannten Bandes durch eine Open-Access-Publikation frei zugänglich. Das Werk ist dennoch insgesamt und in seinen einzelnen Teilen urheberrechtlich geschützt. Das Gesamtwerk bzw. die Einzelbeiträge dürfen nur mit Zustimmung der jeweiligen Autorinnen bzw. Autoren vervielfältigt, übersetzt oder in anderer Weise außerhalb der Grenzen des Urheberrechts genutzt werden. Jede kommerzielle Nutzung ist ausgeschlossen. 


\section{Inhaltsverzeichnis}

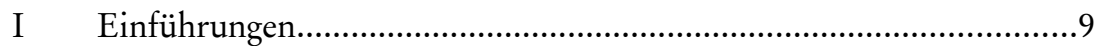

Differenzen über Differenz - Einführung in die Debatten.........................11

Helma Lutz, Norbert Wenning

Die Verwandlung der Philosophie in eine historische Diagnostik

der Differenzen.......................................................................................25

Rita Casale

Differenz und Differenzierung in soziologischer Perspektive

Frank Hillebrandt

Aspekte der angloamerikanischen pädagogischen Differenzdebatte:

Überlegungen zur Kontextualisierung.

Karin Amos

Egalitäre Differenz in der Bildung.

Annedore Prengel

II Disziplintheoretische Zugänge zu Differenz

Feministische Perspektiven auf „Differenz“ in Erziehungsund Bildungsprozessen.

Martina Löw

Das Soziale und die Differenz. Zur (De-)Thematisierung von

Differenz in der Sozialpädagogik.

Susanne Maurer 
Die Rezeption von Differenzdiskussionen in der Vergleichenden Erziehungswissenschaft

Karin Amos

Differenz als Konstitutionsproblem der Sonderpädagogik

Günther Opp, Michael Fingerle, Kirsten Pubr

III Kategorien zur Konstruktion von Differenz. .177

Frauen/Männer, Kinder/Erwachsene.

Rolf Nemitz

Kultur als Differenzierungskategorie.

Thomas Höhne

Differenz als Rechenaufgabe: über die Relevanz der Kategorien

Race, Class und Gender.....

Helma Lutz

An-, Zu- und Ungehörigkeiten Jugendlicher: Herkunft als Auskunft?....231 Clemens Dannenbeck, Hans Lösch, Felicitas Eßer

IV Zur Produktion von Differenz

Dichotome Differenzen und antirassistische Praxis. .251

Rudolf Leiprecht, Susanne Lang

Differenz durch Normalisierung. .275

Norbert Wenning

Die Autorinnen und Autoren. 


\section{Danksagung}

Dieses Buch ist nur zustande gekommen durch die Mitarbeit vieler HelferInnen mit unterschiedlichsten Beiträgen.

Wir bedanken uns insbesondere bei Ingrid Gogolin, Marianne KrügerPotratz, Rudolf Leiprecht, Karl-Ernst Ackermann und den Studierenden des Seminars „Differenzdebatten in der Erziehungswissenschaft“, das im Wintersemester 1999/2000 an der Westfälischen Wilhelms-Universität Münster stattfand, für interessante Hinweise und Kommentare zur inhaltlichen Gestaltung des Buches. Die Autorinnen und Autoren hatten sich mit vielfachen Änderungswünschen unsererseits zu befassen und dabei Ausdauer zu zeigen. Ulrike Fromm, Jeannette Stiller und Bernhard Rosenkötter haben mit viel Geduld die Korrekturen der diversen Versionen des Buches vorgenommen und umgesetzt. Dafür ein besonderes Dankeschön.

Wir hoffen, dass dieses Buch seinen Leserinnen und Lesern Anlass gibt zu heftigen Diskussionen, zu Revisionen und Perspektivwechseln, vielleicht auch zu Widerspruch.

Münster, Sommer 2000

Helma Lutz und Norbert Wenning

Hinweise zur Wiederauflage:

Die erneute Auflage dieses Bandes erfolgt, weil die Verlagsfassung im Buchhandel vergriffen ist und zugleich wiederholt Nachfragen nach dem Text an uns gerichtet werden. Eine vollständige Überarbeitung der Beiträge wäre nach fast zehn Jahren reizvoll, erscheint uns aber als recht aufwändig. Zudem hat sich die Debatte um Differenz in der Zwischenzeit weniger dynamisch entwickelt, als wir es erwartet bzw. erhofft hatten. Darum halten wir die Beiträge dieses Bandes auch in der vorliegenden Form grundsätzlich noch für aussagekräftig. Aus diesem Grund wählen wir eine „Zwischenlösung" und machen die Texte allgemein zugänglich. Die vorliegenden Texte sind seitenidentisch mit denen der ersten Auflage.

Da diese Fassung mit der Schrift UWR-GaramondNo8, die Verlagsfassung aber mit Garamond gesetzt ist, kommt es in manchen Absätzen zu leichten Verschiebungen.

Wir danken allen Beitragenden für ihre Zustimmung zu dieser Form der Veröffentlichung.

Frankfurt am Main, Landau in der Pfalz, Januar 2010

Helma Lutz und Norbert Wenning 


\section{Aspekte der angloamerikanischen pädagogischen Differenzdebatte: Überlegungen zur Kontextualisierung}

\section{S. Karin Amos}

Der Beitrag bindet markante Linien der angloamerikanischen pädagogischen Auseinandersetzung um kulturelle/ethnische „Differenz" an zwei weltsystemtheoretische Zugänge und zeigt Implikationen für die weitere erziehungswissenschaftliche Diskussion auf. Der eine weltsystemtheoretische Zugang ist mit den Namen Immanuel Wallerstein und Etienne Balibar verbunden und befasst sich mit dem Konnex von Nationalstaatlichkeit, Ethnizität und Klassenverhältnissen. Dabei kommt den Institutionen Familie und Schule (als öffentliches Bildungssystem) eine besondere Rolle zu. Es geht u. a. darum, wie gesellschaftliche Kohärenz trotz sozialer Ungleichheit gewährleistet wird. Die US-amerikanische Multikulturalismusdebatte ist hier ein Beispiel für Einsprüche, die den Konnex von Macht, Ethnizität und Kultur in nationalstaatlichen Gesellschaftsorganisationen thematisieren. Anders formuliert: Es geht um Einsprüche, die über die Mobilisierung von Gruppeninteressen auf gesellschaftliche Widersprüche und Ungerechtigkeiten aufmerksam machen, welche gesellschaftlich gelöst werden sollen. Daneben gibt es in der Multikulturalismusdebatte die Tendenz, den Streit auf Kultur, Differenz, Identität und Repräsentation zu fokussieren und auf Forderungen zur Beseitigung von Ungerechtigkeiten zu verzichten. Dann kann es zur unbeabsichtigten Stützung des „Gegendiskurses“ kommen, der Gegenstand des zweiten Teils ist. Hier werden weltsystemtheoretische Untersuchungen zunehmender Standardisierung und Universalisierung der Diskussion über staatlich kontrollierte Erziehung referiert - eine Sichtweise, die modernen Individualismus statt Gruppenzugehörigkeiten zentriert.

Während differenztheoretisch orientierte Zugänge der Critical Pedagogy und der Feminist Pedagogy (sofern letztere die Kritik der Postcolonial Studies an ihrem eigenen Projekt, dem Hinterfragen von Wissenskonstitution, ernst nimmt) sich vor allem mit spezifischen Machtverhältnissen des 
Nationalstaatsmodells befassen, liefert die weltsystemtheoretische, auf Individualismus bezogene Betrachtung Hinweise, dass öffentliche Bildungssysteme ihre gesellschaftliche Integrationsfunktion zunehmend mit ihrer arbeitsmarktbezogenen Allokationsfunktion kurzschließen. Zusammen mit Varianten der Multikulturalismusdiskussion, die essentialistische und kulturalistische Deutungen unterstützen, hat diese Verknüpfung fatale Auswirkungen auf marginalisierte Gruppen der Gesellschaft.

\section{Die US-amerikanischen Culture Wars. Schulen und Curricula als reale und symbolische Kampfarenen}

Die zentrale Funktion von Schule bei der Vermittlung nationaler Werte und darin eingelagerter sozialer Ungleichheiten ist in der amerikanischen Pädagogik gut erforscht. Die Kritik an den schulisch vermittelten Elementen nationaler Kultur ist mit einer Krise der kollektiven Identität verbunden. Die nationale Introspektion (was bedeutet Amerikaner- oder Amerikanisch-Sein) ist traditionell Bestandteil amerikanischer nationaler Identität; die Radikalität der Kritik, die auf den Konstruktionsmodus von Nation zielt und die gesellschaftliche Grenzziehung trifft, ist neu. Die Schwierigkeit ist: Die Anrufung der Individuen als Angehörige einer Nation, die im Staat repräsentiert sind, wird problematisiert; an die Stelle traditioneller nationaler eindeutiger Zuordnungen treten neue, „hybride“ Formen - nach einer Wortprägung der Postcolonial Studies. ${ }^{1}$ Sie lassen Vereindeutigungen nicht mehr zu bzw., es formieren sich Widerstände gegen diese - in den USA schon seit den 1960er Jahren kritisierten - eindeutigen Zuordnungen. Die Widerstände bringen ihrerseits neue Vereindeutigungen hervor (z. B. die Bezeichnung African für in den USA lebende Migranten/Einwanderer aus Afrika, die in anderen Zusammenhängen als African American oder auch Black American bezeichnet werden).

Die folgenden Ausführungen konzentrieren sich auf die amerikanischen pädagogischen Differenzdiskussionen, die die dominante Form der Sozialisation und damit die traditionelle Konstitution nationaler Identität wegen der impliziten Machtstrukturen kritisieren. Zur Verortung dieser Differenzdebatte werden zunächst einige Argumente der Ausführungen Balibars und Wallersteins (Balibar 1998) referiert:

Im Nationalstaat bildet das Volk die Gemeinschaft, die sich in der Institution des Staates wiedererkennt. Da „Volk“ nicht naturwüchsig existiert, muss es sich permanent als nationale Gemeinschaft neu schaffen - vor allem, weil moderne Nationen keine gegebene ethnische Basis haben und nur mit Klassenkonflikten existieren. Balibar folgert daraus, dass es einen Einigungsprozess geben muss, in dessen Verlauf die Individuen in der do- 
minanten Form der nationalen Zugehörigkeit sozialisiert werden. Dieser muss ein Massenphänomen (das Volk muss sich als Volk erkennen) und ein Individuationsphänomen sein. $\mathrm{Ob}$ die ideologische Form dieses Einigungsprozesses als Patriotismus oder als Nationalismus bezeichnet wird, Grundlage ist in jedem Fall, was Balibar „fiktive Ethnizität“ nennt. Erst als „ethnisierte" erscheint die historisch junge Gesellschaftsformation des Nationalstaates als natürliche Gemeinschaft, die durch gemeinsame Herkunft und Interessen gekennzeichnet ist und die erst auf dieser Basis Ursprungsmythen ermöglicht. Die das Volk als fiktive ethnische Einheit konstituierende nationale Ideologie schreibt idealiter jedem Individuum eine ethnische Identität zu und teilt die Menschheit in „Ethnizitäten“ ein, denen ebenso viele denkbare Nationen entsprechen. Somit kann jedes Individuum im Namen des Kollektivs angerufen werden, dessen Namen es trägt.

Balibar thematisiert zwei Konstruktionsmodi der „fiktiven Ethnizität“, die diese als natürlichen Ursprung und nicht als Fiktion erscheinen lassen: „Sprache“ und „Rasse“. Beide sind zumeist verbunden; sie drücken aus, dass der nationale Charakter dem Volk immanent ist. Die Konstitution der fiktiven Ethnizität durch eine gemeinsame Sprache verknüpft die Individuen mit dem fiktiven nationalen Ursprung. Aber erst mit der Schaffung einer einheitlichen Sprache (Nationalsprache) entsteht ein die sozialen Unterschiede relativierendes Ausdrucksmittel einer fiktiven kollektiven Identität. Innerhalb dieses Modus gibt es unterschiedliche Weisen, mit Sprache umzugehen; es geht nur noch um Übersetzungen zwischen Sprachebenen.

Diese Nationalsprache wird in den (Volks-)schulen eingeübt. Die allgemeine Schulpflicht stellt sicher, dass alle Kinder, ungeachtet sozialer Herkunft oder Geschlecht, erreicht werden. Die Schule - das bestätigen alle Weltsystemtheoretiker - existiert weniger für Spezialausbildungen oder zur Reproduktion sozialer Eliten, sondern eher zur Sozialisation der Individuen. Sie ist traditionell eine wichtige Komplementärinstitution zur Familie. Der allgemeine Schulbesuch ist die wichtigste Einrichtung zur Konstituierung von Ethnizität als Sprachgemeinschaft (Balibar 1998, S. 120). Daher ist Balibars Feststellung zentral, dass die Sprachgemeinschaft ideell jeden assimiliert. Kinder von Migranten, bzw. Einwanderer der zweiten Generation, gehen, wenn die schulische Sozialisation erfolgreich war, ebenso selbstverständlich mit Sprache und über Sprache auch mit der Nation um wie die Kinder der Angehörigen der Aufnahmegesellschaft. Die Sprachgemeinschaft ist formal egalitär und ermöglicht allen entsprechend sozialisierten Individuen, an der fiktiven Ethnizität zu partizipieren. Gleichzeitig produziert sie, durch schulische Normen vermittelt, Spaltungen, die sich mit Klassenunterschieden decken. Mit wachsendem Einfluss der Schule auf das Leben wirken sich Unterschiede der sprachlichen und damit der literarischen, kulturellen, technologischen Kompetenz als Kastenunterschiede aus. Sie weisen den Individuen abweichende soziale Schicksale zu. Damit 
ist Schule an sozialer Reproduktion beteiligt - aus unterschiedlichen Perspektiven (etwa Bowles und Gintis oder Bourdieu) ein Dauerthema der Bildungssoziologie.

Sprachliche Gemeinschaften bestimmen wesentlich mit, wie sich Individuen als Subjekte konstituieren. Zur Schaffung einer fiktiven Ethnizität reicht sprachliche Gemeinsamkeit allein nicht aus, weil eine Sprache z. B. verschiedenen Völkern dienen kann. Eine weitere Grenzmarkierung der Nation, und damit der Gesellschaft, ist erforderlich. Balibar bezeichnet sie mit „rassischer Gemeinschaft“. Es analysiert den „symbolischen Kern“ bei der Herstellung fiktiver Ethnizität, der es erlaubt, sich die „rassische“ Einheit als Ursprung und Ursache der historischen Kontinuität eines Volkes vorzustellen. „Rasse“ ist mit Genealogie verbunden. Damit wird eine Generationenkontinuität, ein Verwandtschaftsverhältnis erzeugt, das in der Generationenabfolge eine biologische und geistige „Substanz" weitergibt. Die Vorstellung einer „rassischen Gemeinschaft“ kommt danach erst auf, wenn sich Zusammengehörigkeitsgrenzen auf anderen Ebenen (Sippe, Nachbarschaft, theoretisch auch soziale Klasse) auflösen und imaginär an die Schwelle der Nationalität verlagert werden. Die Nation erscheint dann als große Familie, als amerikanische, türkische, griechische, deutsche ... Familiengemeinschaft (Balibar 1998, S. 123).

Die nationale Sprache und die Vorstellung eines gemeinsamen „rassischen" Ursprungs haben Konsequenzen für den Umgang mit sozialer Ungleichheit. Die „rassische Gemeinschaft“ ethnisiert soziale Unterschiede. Bei der genealogischen Dimension dieser „Fiktion zweiten Grades“ zeigt sich die Verbindung der beiden entlang der Grenzen Ethnizität und Geschlecht konstituierten Ordnungssysteme, denn Generationenabfolge und Familie sprechen auch Geschlechterbeziehungen an. Balibar zufolge (1998, S. 125) entsteht mit den Auflösungen der abstammungsmäßigen Verwandtschaft, der Solidarität zwischen den Generationen und der ökonomischen Funktionen der Familie gerade keine rein individualistische Vertragsbeziehung. Vielmehr entsteht eine Nationalisierung der Familie als Gegenstück zur Identifikation der nationalen Gemeinschaft mit einer symbolischen Verwandtschaft. Da Familie und Volk auf diese Weise eng miteinander verbunden sind, wird das staatliche Interesse an Familie und an Familienpolitik als Teil der Bevölkerungspolitik erklärbar - ein Bereich, der ebenfalls im Rahmen der Nationalstaatenbildung entsteht. Michel Foucault leistete hierzu mit seinen Arbeiten zur „Biopolitik“ einen zentralen Beitrag (den Begriff prägte er für das staatliche Eingreifen in die Privatsphäre). Die amerikanische Sozialwissenschaftlerin Patricia Hill Collins untersuchte dies am Beispiel der unterschiedlichen Konstitution von „schwarzer" und „weißer“ Mütterlichkeit in der US-amerikanischen Gesellschaft und bestätigte die von Balibar und Foucault beschriebenen Strukturen.

Für Balibar ist die Produktion von Ethnizität stets mit einer „Rassisie- 
rung der Sprache“ und einer „Verbalisierung der Rasse“ verbunden. Die Stabilität der nationalen Formation beruht u. a. darauf, dass sie die Staatsbürgerzugehörigkeit der Menschen anderen, vor allem der der sozialen Klasse, überordnet. Dieser Vorrang erfuhr durch die Organisation der Nationalstaaten als Sozial- bzw. Wohlfahrtsstaaten besonderen Nachdruck. Der staatliche Einfluss wurde um Dimensionen erweitert, die zunächst nichtstaatlichen gesellschaftlichen Bereichen vorbehalten waren, den Kirchen etwa oder der familiären Privatsphäre. Die Erweiterung wird zumeist als Beweis für die staatliche Fürsorge des „Vaters“ (Staat) und nicht als Übergriff interpretiert, weil Nationalstaatlichkeit nach dem Modell wechselseitiger Verpflichtung funktioniert - der Staat repräsentiert die Nation und ist für die einzelnen Mitglieder verantwortlich. Diese definieren sich als Solidargemeinschaft und verhalten sich loyal gegenüber dem Staat.

Wallersteins Ausführungen (1998) leuchten dies weiter aus: Er leitet die Kategorien „Rasse“, Nation, ethnische Gruppe ebenfalls aus dem kapitalistischen Weltmarktzusammenhang ab. Einschlägige historisch orientierte sozialwissenschaftliche Darstellungen behandeln „Rasse“ als genetische Kategorie, gekennzeichnet durch eine physische Form, Nation als soziopolitische Kategorie, die mit Grenzen des Staates zusammenhängt und ethnische Gruppe (die den Ausdruck der Minderheit ersetzte) wird zur Kennzeichnung des Kulturellen benutzt, unabhängig von Staatsgrenzen. Wallerstein bezieht „Rasse“ dagegen auf die horizontale Arbeitsteilung zwischen Zentrum und Peripherie, Nation bezeichnet dann souveräne Staaten, die gemeinsam das internationale Staatensystem bilden, und ethnische Gruppe ist eine auf interne Hierarchisierungen der Arbeit bezogene Kategorie. In allen modernen Staaten überlagerte die Ethnisierung der Arbeit Klassenunterschiede. Diese Interpretation erklärt im angloamerikanischen Zusammenhang den prekären Status bestimmter Gruppen. Häufig werden dabei exemplarisch die Afroamerikaner angeführt, weil hier die ökonomische Unterscheidung zwischen Zentrum und Peripherie nach innen verlagert wurde und mit dem Muster der Ethnisierung sozialer Unterschiede zusammenfällt. Für die ungünstigste Konstellation der unterschiedlichen Relationen, die sich sozialräumlich abbildet, wurden die Termini underclass (Auletta 1982) oder ghetto poor (Wilson 1987, 1997, Wacquant 1994) geprägt.

Wird Balibars und Wallersteins Interpretationsrahmen auf die USA angewendet, entsteht folgendes Bild: Die Vereinigten Staaten waren als klassisches Einwanderungsland, als Nation vieler Völker, immer schon das, was aktuell als plurale Gesellschaft gilt, konstituiert aber im Sinne des E Pluribus Unum mit deutlicher Betonung des Unum (eine bekannte Chiffre für dieses Unum ist das Akronym WASP - White Anglo-Saxon Protestant). Unterschiedliche Medien wie Zeitungen, Romane und Schulbücher sowie symbolische Formen wie Gedenktage, Nationalhymne usw. sozialisierten die Individuen in der dominanten Form der Nation. Die Vorstellung einer 
einheitlichen Nation wurde vermittelt, deren innere Bestimmung in der Nationalgeschichte repräsentiert ist, die in den USA die Form einer säkularen Heilsgeschichte annahm. Diese nationale Historiographie, in Balibars Sinn (1998) eine Form der Perpetuierung des nationalen Projekts, hatte entscheidenden Anteil daran, dass die Gesellschaftsmitglieder als Amerikaner „angerufen“ (Althusser) werden konnten. Das amerikanische Schulsystem nahm, obgleich dezentral organisiert, als zentrale Aufgabe die Assimilierung der Einwandererkinder wahr. Als Illustration ein Jahresbericht des Boston School Committee von 1858 zur Beschreibung des staatlichen Erziehungsauftrags für diese Kinder:

„taking children at random from a great city, undisciplined, uninstructed [...] with the inherited stupidity of centuries of ignorant ancestors; transforming them from animals into intellectual beings" (Katz 1995, S. 104).

In den Schulen wurden „Amerikaner gemacht“. In den Schulbüchern fanden sich Einwandererkinder nicht wieder - ethnische und kulturelle Differenz blieb stigmatisiert, den späteren Bemühungen einiger Reformpädagogen zum Trotz.

Hier kann nicht auf unterschiedliche politische und ökonomische Strategien ethnischer Gruppen eingegangen oder die Frage erörtert werden, welche europäischen ethnics zu den "Uneinschmelzbaren" gehören bzw., ob die Unterscheidung in "einschmelzbare“ und "nicht-einschmelzbare“ (meltable and unmeltable) etbnics überhaupt sinnvoll ist. Jedenfalls gingen die Nachkommen europäischer Einwanderer erst im Zuge der schwarzen Bürgerrechtsbewegung der 1960er Jahre auf die Suche nach ihren Wurzeln, was sich dann in Bindestrich-Bezeichnungen der Reethnisierungspolitik ausdrückte: Irish-American, Italian-American, Polish-American usw. Den materiellen Hintergrund bildete der Kampf um Ressourcen; unterschlagen wurde, dass der Anlass des schwarzen Kampfes eine rigidere Exklusion war, als europäische Einwanderer sie je erlebten. Zwar gab es über mehrere Generationen eine soziale Hierarchie europäischer Einwanderer und eine deutliche Ethnisierung des Arbeitsmarkts, die sich in den Großstädten auch sozialräumlich abbildete, aber Einwanderer beteiligten sich am Ausschluss der Schwarzen, wie auch die Gewerkschaftsgeschichten zeigen.

Ausgangspunkt für die aktuelle Diskussion um racial bzw. ethnic difference war die von der Schwarzen Bürgerrechtsbewegung artikulierte Forderung nach umfassender gesellschaftlicher Inklusion. Die gesellschaftspolitische Zielsetzung hieß zunächst Integration, aber Auseinandersetzungen verdeutlichten, dass kein Konsens herrschte. Die Black Panthers wollten eine grundlegende Neuorganisation des Staates, die Schwarzen Separatisten eher Schwarzen Nationalismus und afroamerikanische Autarkie - mit Referenz auf ein eigenes Symbol- und Repräsentationssystem. Diese Richtungen vertraten u. a. Malcolm X und Stokely Carmichael. Martin Luther King Jr. repräsentierte eher den Teil der schwarzen Gemeinde, der auf Ei- 
nigung und Verständigung mit den Weißen setzte und der in seinen Reden und Predigten zahlreiche Verbindungen zur nationalen Metaphorik herstellte, man denke an seine berühmte „I Have a Dream”-Rede. In der religiösen Orientierung wird der Bruch besonders augenfällig: Für das durch und durch christlich definierte nationale Selbstverständnis der USA war die Hinwendung eines Teils der Black Community zum Islam provokant. Diese in den 1960ern einsetzende Entwicklung war Auslöser weiterer Differenzierungen in den Afrozentrismus (eine der Hauptformen ethnischer Identitätspolitik) und in die aus der Rezeption des französischen Poststrukturalismus hervorgegangenen Debatten um radikale Alteriät.

Die Analyse des Zusammenhangs gruppenspezifischer Positionierung mit gesellschaftlichen Machtverhältnissen hatte für die Erziehungswissenschaft zur Folge, dass die Beziehung von gruppenspezifischem Schulerfolg und sozialer Benachteiligung, das Verhältnis von Identität und Repräsentation (vgl. das Programm der Cultural Studies), auf die (bildungs-)politische Agenda kam. Kompensatorische Erziehung ging noch von der Farbenblindheit der Schulen aus und führte gruppenspezifischen Misserfolg auf das Sozialisationsumfeld der Kinder, d. h. die Familien (vor allem die als dysfunktional diskreditierten schwarzen Familien) zurück (vgl. Moynihan 1965), dann folgte eine Untersuchung der in die Schulstrukturen eingeschriebenen Diskriminierungsmechanismen. Neben dem individuellen kam vor allem der institutionelle Rassismus in den Blick; Curricula und Schulbücher, Lehrerausbildungspolitik und Lehrerbeschäftigung wurden zu Untersuchungsgegenständen.

Noch in den 1960er Jahren gab es erste Untersuchungen insbesondere von Schulbüchern, die die nationale Identität tradieren und konstruieren: Civics- (Staatsbürgerkunde-), Sozialkunde- und Geschichtsbücher. Bis in die frühen siebziger Jahren gab es zugleich heftige Auseinandersetzungen um Black English. Es ging um die Frage, ob dies eine eigene Sprache, ein Dialekt oder "falsches" Englisch sei: Hier liegen die Wurzeln der aktuellen Ebonics-Debatte. Bikulturelle und bilinguale Erziehung traten auf den Plan - ein Indiz, dass sich das öffentliche Schulsystem der USA auf demographische Veränderungen und die veränderte Wahrnehmung sowie Bewertung gesellschaftlicher Pluralität einzustellen begann. Dies übte maßgeblichen Einfluss auf die Diskussionen des an Colleges und Universitäten (aber auch an high schools) implementierten Literaturkanons aus. Kritisiert wurde die Fixierung auf westliche Inhalte und die Nicht-Berücksichtigung anderer Stimmen und Standpunkte. Dinesh D'Souza, E. D. Hirsch und Allan Bloom sind mit der konservativen Position verbundene Namen, der sich Arthur M. Schlesinger Jr. mit der Frage: E Pluribus Unum? bezugnehmend auf das Motto des Großsiegels der Vereinigten Staaten anschloss.

Mit den sich in Curricula und Schulbuchinhalten spiegelnden Machtverhältnissen und Positionierungen befasste sich vor allem die Kritische 
Pädagogik ${ }^{2}$, ein Bereich, der nur verkürzt als bildungssoziologisch zu beschreiben ist. Anregungen bezieht sie aus den Cultural und Feminist Studies sowie aus Teilen der deutschen kritischen Theorie, den Arbeiten Pierre Bourdieus sowie von Frantz Fanon und Paulo Freire. Ziel dieses Theoriemixes ist die Erforschung des Zusammenhangs von Wissen und Macht sowie der Reproduktion von symbolischem und kulturellem Kapital: kurz, die Untersuchung von Grenzverläufen und Exklusionsmechanismen im Bildungsbereich. Der französische Poststrukturalismus (vor allem Foucaults Mikrophysik der Macht und Derridas Dekonstruktivismus) spielt dabei eine wichtigere Rolle als die quantitative Auswertung großer Datenmengen. Den Ergebnissen zahlreicher mit den Konzepten der Kritischen Pädagogik arbeitender Analysen zur Curriculum- und Schulentwicklung, den ethnographischen Studien zu Lehrer- und Schülerinteraktionen und den begleitenden kritischen Reflexionen von Schulreformen ist zu verdanken, dass sich „an den Rändern“ und „in den Zwischenräumen“ neue Formen individueller und kollektiver Identität (gebunden an neue Repräsentationsund Artikulationsmöglichkeiten) bilden konnten - die Formulierungen deuten schon den zentralen Einfluss der Cultural und der Postcolonial Studies an. ${ }^{3}$ Die in der Nationalstaatskonstruktion eingeschriebenen Herrschaftsverhältnisse sollen durch Analysen und Kritik offengelegt werden. Die Kritik dieser Herrschaftsverhältnisse ist eine der Wurzeln für die im Kontext der Postmoderne-Diskussion erfolgte Neudefinition des Nationalstaats als "Gemeinschaft von Gemeinschaften“.

Dies hatte Folgen für die Organisation des öffentlichen Schulsystems. Mit den Wirkungen neuer Organisationsformen des Bildungssystems auf bestimmte ethnische Gruppen befasst sich daher auch die traditionelle Schulforschung. Seit den 1960er Jahren erhoffen sich nach amerikanischem Verständnis liberale und radikale Bildungspolitiker und -forscher von einer Schulreorganisation durch Community Action, Deregulierung und Schulautonomie die Verwirklichung von Chancengleichheit für benachteiligte Gruppen durch höhere Bildungserfolgsquoten. Kernpunkt der Attacken während der Bildungsoffensive der Sechziger waren die schwerfälligen Bü-

2 Dazu gehören unterschiedliche Bereiche; sehr produktive kritische Pädagogen sind Henry Giroux (z. B. 1983, 1992, mit Peter McLaren 1989) ebenso wie Michael W. Apple: (1982, 1986, 1993, mit Weis 1983, mit Christian-Smith 1991) und Martin Carnoy (1984, mit Henry M. Levin 1985)

3 Einige einschlägige aktuelle Veröffentlichungen zu den Cultural Studies: Hörning/Winter 1999, Bromley/Götllich/Winter 1999, Engelmann 1999. Die englischsprachige Literatur ist umfangreich und divers, deshalb hier nur der Verweis auf den voluminösen Reader von Grossberg/Nelson/Teichler 1992. Für den Bereich der Postcolonial Studies haben Bronfen/Marius/Steffen 1997 eine erste Auswahl für die deutschsprachige Leserschaft herausgegeben; Beachtenswert für diesen Bereich wie für die Critical Pedagogy sind die Beiträge in der Zeitschrift: Das Argument. Aus der Vielzahl der englischsprachigen Beiträge zum Stichwort Postcolonial Theory/Studies hier nur zwei Hinweise: Ashcroft/ Griffiths/Tiffin (1995) und die hervorragende Einführung von Loomba (1998). 
rokratien großer urbaner Schulsysteme, in denen einige Minderheiten, ablesbar an niedrigen Bildungserfolgsquoten, besonders benachteiligt waren. Die Demokratisierung durch erhöhte Kontrolle seitens der Community sollte die Bedürfnisse der SchülerInnen eher zum Tragen bringen und deren Bildungsprobleme besser lösen. Schuldezentralisierung als Teil einer umfassenden sozialen Forderung nach Inklusion marginalisierter Gruppen beinhaltete daher die Vorstellung von Machtverteilung auf neue, außerhalb des traditionellen politischen Systems gebildete Interessenvertretungen. Veränderungen der Entscheidungsprozesse in den Bürokratien sollten die Inklusion benachteiligter Teile der afroamerikanischen Gemeinde fördern.

Die radikalste Institutionenkritik war die Forderung nach Deinstitutionalisierung (Goffman 1961, Becker 1963). Nach deren Grundprämisse bringen Institutionen erst die Menschen hervor, die sie ändern sollten. Diese Kritik totaler Institutionen wurde in den 1970ern auf Schulen übertragen das unterstützte politisch den moralischen Impetus der Reformideen der späten Sechziger (Demokratie und Inklusion). Ob diese Interpretation für Goffmans Ansatz angemessen ist, wird hier nicht weiter verfolgt. Es ging um die Alternative zwischen zwei Modellen: die offene Institutionsform, durch die Gemeinschaft (Community) kontrolliert, oder die geschlossene Institutionsform, kontrolliert durch das Prinzip der Professionalität. Die maximale Partizipation der Gemeinschaft bedeutete eine Verpflichtung auf das „Ermächtigungskonzept“ (Empowerment). Dieses Prinzip lag den Community Action-Programmen zugrunde, wichtigen Bestandteilen des umfassenden „Kriegs“ gegen Armut (dem War on Poverty der Johnson Administration). Bei den vergangenen Bildungsreformprogrammen wie bei den aktuellen, die ebenfalls auf die Stärkung des Community-Einflusses zielen, ist grundlegend zu unterscheiden: Staatliche Reformen beziehen sich zumeist auf die mit der progressiven Erziehungsbewegung und dem Namen John Dewey verbundene amerikanische Bildungstradition, d. h. auf den Einbezug der Community und die Professionalisierung der Bildung. Die Communities können dabei mitreden, aber nicht entscheiden. Die Community-Action-Programme der Sechziger und die mit dem Empowerment-Prinzip arbeitenden aktuellen Programme verfolgen dagegen eine Übertragung der Entscheidungskompetenzen auf die Gemeinden.

Besonders kritisch zu den auf Community-Action-Forderungen zurückgehenden Schuldezentralisierungen in New York steht Fred Siegel (1997). Er stellt heraus, was oft unthematisiert bleibt: die historisch gewachsene Aufteilung des öffentlichen Dienstes in ethnische fiefdoms, feudale „Lehnsbezirke“ - eine metaphorische Kennzeichnung vormoderner Strukturen der Aufteilung von Territorien und Arbeitsbereichen - unter Ausschluss der Schwarzen. Die Ausgaben für New Yorks Schulen wurden, in Folge der schwarzen Bürgerrechtsbewegung, zwar drastisch erhöht, und ein Großteil der Mittel kam den Slums zugute. Da die verschiedenen Interes- 
senvertretungen in unversöhnliche Gegensätze gerieten, wurde eine effektive Schulpolitik aber verhindert. Neben dem Konflikt zwischen Community und Schulbürokratie entstand in den 1960ern ein weiterer, bis heute andauernder Streit, der zwischen „weißen“ und „schwarzen“ Lehrern. Die Black Power Bewegung und der schwarze Nationalismus sahen in jeder Integration eine Assimilation an „weiße“ Werte und lehnten sie daher ab. Der Ausweg bestand in der Etablierung eigener, afrozentrischer Schulen. Siegels kritische Einschätzung des Kompromisses bei der Neuorganisation des öffentlichen urbanen Schulsystems wird von wissenschaftlichen Untersuchungen zur Schuldezentralisierung in New York und Chicago (etwa Lewis/Nakagawa 1995) geteilt: die Bildungschancen von Minderheiten wurden nicht erhöht. Die Erwartung, mit der Schuldezentralisierung einen entscheidenden Beitrag zur Lösung des American Dilemma ${ }^{4}$ zu leisten, erfüllte sich nicht. Die Weißen behielten ihre Hegemonie, indem sie den Schwarzen die Kontrolle über Institutionen wie dem öffentlichen Schulsystem der Inner Cities überließen. Analysen der Modelle verschiedener Städte ergeben: Weder Integration noch Schulerfolg machten nennenswerte Fortschritte. Einmal etabliert, dient die dezentralisierte Schule ebenso zur Aufrechterhaltung des Status quo wie die zuvor kritisierte traditionelle.

Die Schulen bleiben getrennt und ungleich. Der Rassenkonflikt, der sich an der Aufhebung der Segregation in den Schulen entzündete, wurde in einen Konsens über die antidemokratische Natur öffentlicher Bürokratie, dem Organisationsprinzip der Schulen (Tyack 1974, Katz 1971) und die Bedeutung von Community Empowerment transformiert.

Eine Zusammenfassung der Prämissen der Community-Action-Programme und der tatsächlichen Entwicklungen:

1. Eine effektive Schulbürokratie führt automatisch zu größerer sozialer Mobilität. Diese Annahme unterschätzte die Wirkung von in anderen gesellschaftlichen Bereichen und Institutionen eingeschriebenen „Rasseund Klassenstrukturen" (Omi/Winant 1994).

2. Größere Beteiligung der Eltern und Gemeindemitglieder führt zu höherer Schulleistung. Untersuchungen (z. B. Fine 1993) konnten hierzu keinen Zusammenhang feststellen.

3. Macht und Autorität sind durch gesetzgeberische Maßnahmen leicht umzuverteilen und zu übertragen. Diese Annahme unterschätzte das Beharrungsvermögen von bestehenden Ressourcenverteilungen und bürokratischen Verteilungsmechanismen.

Auf dem Empowerment-Prinzip ${ }^{5}$ beruhende Schuldezentralisierungsmodelle

$4 \quad$ Von Gunnar Myrdal 1944 geprägter Ausdruck für die Unvereinbarkeit von Rassensegregation mit den amerikanischen Prinzipien von Gleichheit und Demokratie. Empowerment ist auch ein zentraler Begriff der Critical Pedagogy. Auf den jeweiligen Verwendungs- und Bedeutungszusammenhang ist sorgfältig zu achten. 
sind weniger erfolgreich als nach dem Enablement funktionierende. Im letzteren Ansatz beraten Eltern mehr, sie entscheiden nicht. Der Einfluss und Handlungsspielraum des Lehr- und Verwaltungspersonals wird hier gestärkt. Häufigste Form des Enablement-Ansatzes ist das School-Based Management (SBM). Dieser Ansatz will die Reformmöglichkeiten innerhalb des bestehenden bürokratischen Rahmens besser nutzen.

Während der 1980er Jahre legitimierte die Machtverteilung die Trennung von „Schwarzen“ und „Weißen“. Jetzt zeichnet sich jedoch ab, dass die Schuldezentralisierung zur Spaltung zwischen schwarzer Mittelklasse und weniger privilegierten Afroamerikanern führt. Der dieser Spaltung zugrunde liegende Konflikt hat folgenden Hintergrund (womit die an staatliche Unterstützung appellierende Strategie der Bürgerrechtsbewegung am deutlichsten unterstrichen wird): Die schwarze Mittelklasse ist u. a. Resultat der Gleichstellungsmaßnahmen der Affirmative-Action-Programme, die auch und gerade im öffentlichen Dienst das Angebot von Arbeitsplätzen für Minderheiten vergrößerten. In den Schulen der Inner Cities sind Lehrende und Verwaltungspersonal großer urbaner Schulbürokratien Angehörige der gleichen Minderheiten, deren Kinder in den bis zu 6.000 Schülern aufnehmenden Schulsystemen unterrichtet werden - und häufig scheitern. Die Schulkritik führte, wie erwähnt, die Bildungsmisere besonders auf bürokratische Überregulierung zurück, der vorgeworfen wurde, an den Bedürfnissen der Schüler und der Communities vorbei zu planen. Für den Empowerment-Ansatz ändert die Besetzung der Verwaltungsstellen mit Minderheiten nichts, da die Strukturen „weiß“ bleiben.

Aber auch größere Mitsprache und Einflussnahme der Communities lösen die Probleme nicht: Die Tragik des heutigen Zustands der Schulen in vielen amerikanischen Metropolen ist, dass sich Angehörige der gleichen ethnischen Gruppen feindlich gegenüberstehen und dass - obwohl weniger sichtbar als zu Beginn in den 1960ern - die Ideologie der Dezentralisation die „Rassenhierarchie“ stützt. Weniger sichtbar deswegen, weil sich die Weißen fast völlig in die Vorstädte zurückzogen. „The Result is an educational system that supports the racial hierarchy" (Lewis/Nakagawa 1995, S. 18, vgl. Siegel 1997, Kap. 3 und Gitlin 1995). Auch die Erfolge (z. B. Meier 1995) ändern am weitgehenden Scheitern der Reformen in den großen innerstädtischen Schulsystemen wenig. Besonders betroffen von den sich verschlechternden Bildungschancen sind die in allen relevanten gesellschaftlichen Bereichen stark marginalisierten Afroamerikaner in den Ghettos und die Latinos in den Barrios. Indiz ist der aktuelle Streit um Black English: Unter der Überschrift: Non-Standard English wurde diese Kontroverse bereits in den frühen 1970ern verhandelt; Anfang der 1990er tauchte sie unter dem Stichwort: Ebonics (ein Terminus aus den frühen Siebzigern, der damals aber keine Verbreitung fand) wieder auf. Die Befürworter erkennen die Grundperspektive von Ebonics an, Black English als eigene, regelgeleite- 
te Sprache zu sehen und nicht als falsches Englisch zu kritisieren, und halten sie für einen großen Fortschritt. Die Sensibilisierung der Lehrer, die mit einer hauptsächlich Ebonics sprechenden Schülerpopulation zu tun haben, begrüßen sie als ersten wichtigen Schritt zum Verständnis und zur Wertschätzung ihres kulturellen Hintergrunds (zusammenfassend Perry/ Delpit 1998). Für die Gegner von Ebonics ist die Institutionalisierung eines Schulfachs Ebonics, zusammen mit anderen Elementen eines afrozentrischen Curriculums, ein gefährlicher Schritt, weil solche Maßnahmen das Auseinanderdriften der Lebenswelten fördern und die Inklusionschancen der Marginalisierten in den Inner Cities so weiter abnehmen. Diese Überlegungen sind mit Bourdieus Theorie sozialer Reproduktion kompatibel; der bildungssoziologische Zusammenhang verweist auf die zentrale Bedeutung von kulturellem und symbolischem Kapital - u. a. am geringen Tauschwert gruppenspezifischer Wissensbestände auf dem Arbeitsmarkt ablesbar.

Aktuelle Ghettoforschungen unterstreichen, dass Sprachkompetenz in Standard Englisch sozusagen Einstellungsvoraussetzung ist. Dies gilt besonders auf dem postfordistischen Arbeitsmarkt, der Beschäftigungsmöglichkeiten für junge Ghettobewohner vor allem auf unteren Stufen der Dienstleistungsökonomie bietet. Loic Wacquant beurteilt am Beispiel des Chicagoer urbanen Schulsystems unmissverständlich den desolaten $\mathrm{Zu}$ stand der Schulen in den Ghettos:

"no organization is more emblematic of the degree of institutional debilitation and political abandonment suffered by Chicago's ghetto than the public schools" (Wacquant 1994, S. 428).

Damit kommt er zur gleichen Einschätzung wie Lewis und Nakagawa (1995), die Chicagos Schulsystem als „Reservat für arme Minoritäten“ beschrieben. Die in ethnographischen Studien dokumentierten schlechten Schulerfahrungen und -erinnerungen der "Schulabbrecher" zeigen, dass der (Tausch)Wert formaler Bildung in den Ghettos und Barrios wohl erkannt wird; alle Reformen setzen die Ansätze und Integrationsüberlegungen aber bisher nicht in größerem Maße in messbar höhere Schulerfolgsraten um.

Abschließend ein Beispiel, das veranschaulicht, wie um Schulbuchinhalte gerungen wird und wie sehr dabei Fragen der Gruppenrepräsentation im Vordergrund stehen. Bildungsaktivisten und -theoretiker versprechen sich von Curriculumreformen, vor allem durch Einführung neuer Ansätze und Fächer oder durch radikale Schulbuchrevisionen, eine höhere gesellschaftliche Inklusionsrate marginalisierter Gruppen. Das verdeutlichte insbesondere die Ebonics-Kontroverse. Schauplatz des Beispiels ist Oakland; die Protagonisten sind: Oakland School Board und eine vom Verlag Hough ton Mifflin in Auftrag gegebene Schulbuchserie zur amerikanischen Sozialkunde und Geschichte für die Elementarstufe bis zur achten Klasse. Die Entscheidung über die Annahme neuer Schulbücher ist in Kalifornien ein langwieriger, viele Instanzen involvierender, aber demokratischer Prozess; 
die letzte Stufe ist die Abstimmung durch den School Board. Die Schulbuchserie wurde mit der Maßgabe in Auftrag gegeben, die Pluralität und Diversität der amerikanischen Nation widerzuspiegeln. Zunächst schien es, als verwiesen die im Entscheidungsprozess vom School Board beklagten Missrepräsentationen und Stereotypen nur auf einen längeren Revisionsprozess. Die Diskussionen zeigten aber ein anderes Problem: die Unmöglichkeit, alle Standpunkte zu berücksichtigen und trotzdem eine nationale Geschichte zu schreiben (vgl. vor allem Gitlin 1995).

\section{2 „Weltkulturmodelle" und Nationalstaat}

In weltsystemtheoretischer Perspektive fragte eine Gruppe von Sozialwissenschaftlern, u. a. John W. Meyer, Francisco Ramirez und John Boli, nach den Ursprüngen und der Expansion staatlich institutionalisierter Erziehungssysteme sowie in neueren Publikationen in makrophänomenologischer Perspektive und unter Nutzung aktueller Theorien sozialer Institutionen nach der Ausprägung eines „ortlosen“ Weltkulturmodells. ${ }^{6}$ Diese Forschungsrichtung sieht "Weltmodelle" als zunehmend universalisiert und standardisiert an und hält sie für das „Skript“, das die Rolle definiert, die von den einzelnen Akteuren - den Nationalstaaten - auszufüllen ist (der Anklang an Goffman ist hier beabsichtigt).

„Ortlose Weltmodelle“ können auffällige Charakteristika moderner Gesellschaften erklären, die so auf den Nenner zu bringen sind: struktureller Isomorphismus angesichts enormer Unterschiede. Der strukturelle Isomorphismus bezieht sich auf Phänomene der fast ausnahmslos nationalstaatlichen Organisation moderner Gesellschaften, der strukturellen Gemeinsamkeiten ihrer internen Organisation sowie der ähnlichen Reaktion auf Veränderungen. Die „Weltmodelle“ gehen auf globale kulturelle und assoziative Prozesse zurück. Assoziativ im Sinne der These Tocquevilles, die er im Rahmen seiner in der ersten Hälfte des 19. Jahrhunderts veröffentlichten, komparativen Demokratieanalyse vertrat, dass den freiwilligen Vereinigungen und Zusammenschlüssen zentrale Bedeutung beim Verständnis politischer und sozialer Prozesse beizumessen sei. Das gleiche Prinzip sehen Meyer, Ramirez und Kollegen auf globaler Ebene wirken. Sie konstatieren, dass die Zielsetzungen und Programme, die in den Weltmodellen für die einzelnen Gesellschaften formuliert werden: Gleichheit, Achtung der Menschenrechte, Abschaffung diskriminierender Praktiken, Institutionalisierung von Wohlfahrtssystemen, soziale Gerechtigkeit, Schutz individueller Rechte, die Autorität gesetzlich geregelter Kontrollsysteme, aber auch die Verpflichtung auf aktive staatliche Unterstützung

Auch hierzu eine Auswahl: Meyer/Boli/Thomas/Ramirez 1997, Ramirez/Rubinson 1979, Meyer/Kamens/Benavot/Wong 1992. 
sozioökonomischen Fortschritts bis hin zur globalen Rolle des Umweltschutzes, in hohem Maße rationalisiert und standardisiert vorliegen.?

Das Konzept des ortlosen Weltkulturmodells illustrieren sie mit dem Beispiel einer neu „entdeckten“, noch nicht in die Weltgesellschaft integrierten Inselgemeinschaft. Die Inselgemeinschaft wird sich nationalstaatlich organisieren und in entsprechende Teilsysteme (Wirtschaft, Recht, Bildung usw.) ausdifferenzieren. Sie wird sich eine Verfassung geben und nationalstaatliche Ziele formulieren - dieses „Programm“ wird entscheidend durch die „Weltkultur“ geprägt sein. Prägnant wird auch das Bildungssystem sein. Die Organisation (Gliederung in Jahrgangsklassen, Unterscheidung in Primar-, Sekundarstufe und tertiären Hochschulbereich, standardisierte Lehrerbildung, Zertifikationssystem, Entwicklung eines Curriculums) sowie die in die institutionalisierte Erziehung gesetzten Erwartungen werden „standardisiert" sein. Ein Zusammenhang zwischen individueller und nationaler Entwicklung, zwischen individuellem und nationalem Fortschritt wird angenommen, nationale Bildungsstatistiken werden erhoben und dem internationalen Bildungsvergleich und der internationalen Leistungsmessung Bedeutung beigemessen werden. Als Beleg für das erwartbare Vorgehen in diesem hypothetischen Fall sehen die Wissenschaftler die tatsächliche internationale Entwicklung. Nach dem Zweiten Weltkrieg, mit Beschleunigung in den 1960er Jahren, nahm die Zahl der Nationalstaatsbildungen $\mathrm{zu}$ - ein deutlicher Beleg für die Universalisierung dieses gesellschaftlichen Organisationsmodells. Daneben wurden die Signifikationssysteme standardisiert. Die weltweite Betonung des internationalen Schulleistungsvergleichs und der Bedeutung von Erziehungssystemen für individuellen wie nationalen Fortschritt - wozu in jüngster Zeit, nicht nur in westlichen Staaten, die Forderung nach Hochschulzugang für Frauen zählt - zeigt dies. Als Illustration für die Bedeutung, die dem Zusammenhang von "Geschlecht und Bildung" beigemessen wird, dienen Sondernummern erziehungswissenschaftlicher Zeitschriften. ${ }^{8}$

Danach dient die den Weltmodellen entnommene Argumentation dazu, Agenden für lokale Handlungen zu definieren und zu legitimieren.

Fragen ungleicher Machtverteilung thematisieren sie nur am Rande. Sie untersuchen die Semantik, nicht die gesellschaftliche Realität. Sie wollen die Sogwirkung veranschaulichen, die dieses Weltkulturmodell auf die einzelnen Nationalstaaten ausübt.

$8 \quad$ Allein in den zur Vergleichenden Erziehungswissenschaft zu zählenden Zeitschriften: die Sondernummer: The Education of Women. L'Èducation des Femmes. Frauenbildung in der vom UNESCO Institut Hamburg herausgegebenen International Review of Education 1973, Women and Education in the Third World, Comparative Education Review vol. 24 (June 1980) mit Bibliographie, International Review of Education, Women and Education vol. 33, No.4 (1987), darin Sandra Ackers Beitrag. Stellvertretend für den Zusammenhang nationaler Bildungspolitik mit der Inklusion von Mädchen und Frauen im Bildungssystem das australische Beispiel: Policy for the Education of Girls: A Draft Action Plan 1992: Commonwealth Schools Commission, The National Policy for the Education of Girls in Australian Schools, Canberra 1987, Connors/Mc Morrow 1988. 
Weltmodelle beeinflussen nachhaltig Strukturen und politische Programme der Nationalstaaten; allerdings lässt die Aneignung von Weltkulturmodellelementen keine zwingenden Rückschlüsse auf die Praxis zu. Nationalstaaten sind dabei eher loosely als tightly coupled systems. Oft stehen beispielsweise Versprechungen egalitärer Staatsbürgerschaft in Widerspruch zu diskriminierenden Praktiken. Dafür gibt es verschiedene Gründe: das Weltkulturmodell ist hochgradig idealistisch und kann nicht en bloc implementiert werden, manche Elemente sind leichter zu implementieren als andere. In jedem Fall kann man Programme schneller formulieren - etwa zur Gleichstellung der Geschlechter - als umsetzen. Das Beispiel ist bezeichnend: die weltweite Verpflichtung auf Gleichstellung und Integration von Frauen in Institutionen höherer Bildung gehört zu den Beobachtungen, die die Annahme eines „Weltmodells“ rechtfertigen. Andere Erklärungsversuche, die Bildungsintegration von Frauen etwa mit der Entwicklung westlicher Gesellschaften in Verbindung bringen, können nicht begründen, warum diese programmatische Expansion weltweit auftritt. ${ }^{9}$

Die durch territoriale Grenzen, Souveränität, demarkierte Bevölkerung usw. gekennzeichneten Nationalstaaten sind - aus weltsystemtheoretischer Perspektive (vgl. Balibar/Wallerstein 1998) - in erster Linie das Produkt exogamer Kräfte, die aus der Dynamik des, zunächst auf Europa beschränkten, Interstaatensystems entstanden. Nationalstaaten sind „Schauspieler", die sich an die Vorgaben eines Skripts halten. Dieses beinhaltet an zentraler Stelle die Institutionalisierung von „Massenbildungssystemen“. Die Formulierungen nationaler Bildungsziele zeigen die postulierte Verbindung zwischen individueller und kollektiver Entwicklung.

Robert Fiala und Audri Gordon Lanford (1987) fragten nach dem Einfluss der mit Bildung und Erziehung assoziierten Elemente einer Weltkultur auf unterschiedliche Länder. Um zu erkunden, wie die Bildungsideologie des Weltsystems Einfluss auf einzelne Staaten ausübt, analysierten die Autoren aus einem Pool von 125 Ländern für den Zeitraum von 1950 und 1970 die Daten, die Auskunft über die postulierten Bildungsziele geben. Grundlage waren internationale Erhebungen wie z. B. das World Survey of Education. Im Zentrum ihrer Überlegungen steht, was sie globale „Kultur“ nennen. Dabei geht es weniger um normative Komponenten, die bestimmte Verhaltensweisen vorschreiben, als um kognitive Elemente, die den Rahmen für eine bestimmte Sicht von Welt vorgeben. Aus globaler Perspektive ist Kultur ein Set von Annahmen bezüglich Natur und sozialer Welt, somit ein wesentliches Element der Konstruktion und Rekonstruktion sozialen Lebens. Dazu gehören formale Legitimierungen und Ideologien, die wiederum den Aufbau von Organisationen und anderen formalen Institutionen beeinflussen. In Bezug auf die Bildungsvorstellungen identifi-

Zur weltweiten Expansion von Gleichstellungsforderungen vgl. u. a.: Bradley/Ramirez 1996, Ramirez/Weiss 1979. 
zieren die Autoren folgende Elemente: Das Individuum ist die zentrale Einheit sozialer Handlungen. Extensive Sozialisation ist notwendig, um die Potentiale der Individuen zu entwickeln und sie auf ihre Rolle als Erwachsene vorzubereiten. Insofern sind Sozialisationstheorien Teil der Ideologie des Individualismus. Der Nationalstaat ist Hauptdefinator gesellschaftlicher Werte, des Fortschritts und der Entwicklung. Aufgrund des postulierten Zusammenhangs von individueller und nationaler Entwicklung übernimmt der Staat Verantwortung für die Individuen; umgekehrt sind sie durch die Staatsbürgerschaft an den Nationalstaat gebunden. Diese Bindung übersetzt individuelle Handlungen und Fähigkeiten in sozialen Fortschritt. Institutionalisierte Messungen in Form von standardisierten statistischen Erhebungen, primär auf ökonomische und technische Entwicklung, Alphabetisierungsrate und Bildungsexpansion bezogen, dienen der individuellen Evaluation ebenso wie der nationalen. Diese Evaluation ist wiederum Grundlage zur Einschätzung des eigenen Rangs im internationalen Kontext.

Diese Weltkulturelemente geben Rechenschaft über die soziale Welt, in der die Individuen handeln müssen, um die vorgegebenen Ziele zu erreichen. Die formalste Art der Rechenschaft findet sich in Humankapitaltheorien, die Erziehung als ertragversprechende Investition auffassen.

In diesen Analysen spielt neben dem Nationalstaat das Individuum als soziale Konstruktion eine herausragende Rolle. Deshalb wird dieser Aspekt mit Meyers (1986) Überlegungen zum Verhältnis von Individualismus und Sozialisation noch einmal aufgenommen. „Individualismus“ ist hier das Kernkonzept für die Relation zwischen individueller und nationaler Entwicklung. Ursprünglich war Individualismus Teil des westlichen ideologischen Systems; in jüngerer Zeit wurde der Begriff aber bedeutend für das Selbstverständnis moderner Gesellschaften. Mit „Individualismus“ sind zwei zentrale Annahmen verbunden, die auch Fiala und Lanford (1987) thematisierten: menschliche Akteure müssen systematisch sozialisiert werden, diese Sozialisation geschieht am effektivsten in modernen Massenerziehungssystemen. Die Sorge um eine den gesellschaftlichen Bedürfnissen angemessene Konstruktion von Individuen zählt zu den intrinsischen Komponenten moderner Sozialstruktur. Sie entspricht - wenn auch auf anderer Ebene - Bürokratien und großräumigen Wirtschaftssystemen. Individualismus meint hier also eine institutionelle historische Konstruktion und nicht die persönliche Organisation von Erfahrungen. Als historische Konstruktion ist Individualismus einem ständigen Rekonstruktionsprozess unterworfen. Leitet man „Individuum“ von der Ideologie des Individualismus $\mathrm{ab}$, ist es gerade nicht Gegenpol zu den unpersönlichen Kräften bürokratischer Staaten und anonymer Wirtschaftsmärkte. Es ist vielmehr Teil des Systems von Markt und Staat. Die Expansion des Individualismus geht einher mit der des Staates und mit der seit dem Ende des Zweiten Weltkriegs $\mathrm{zu}$ verzeichnenden Ausbreitung des westlichen ideologischen Systems. Au- 
ßerdem ist der Individualismus eine öffentliche und keine private Doktrin. Sie beinhaltet, dass die Gesellschaft in den Verhaltensweisen und Entscheidungen des Einzelnen wurzelt und sich durch den Nutzen für die einzelnen Mitglieder legitimiert.

Diese weltsystemtheoretische Perspektive legt nahe, dass die Bedeutung der Semantik der Internationalität auch und gerade im Bereich institutionalisierter Bildung für die einzelnen Nationalstaaten eher zu- als abnehmen wird. Die öffentliche Aufmerksamkeit für internationale Schulleistungsvergleiche ist hierfür ein wichtiger Indikator; über Qualitätssicherung, Effizienz und Autonomie wird fast immer mit Verweis auf die internationale Diskussion geredet. Berichte über „Toyota-Schulen“ gelten auch in der hiesigen Diskussion als Beispiele für die Verschränkung von Schul- und Wirtschaftssystem. Die Kennzeichnung „national“ verdeckt, dass die Auseinandersetzung über nationale Curricula stärker von internationalen Vorgaben als von inneren gesellschaftlichen Entwicklungen geprägt ist.

Das Beispiel „Toyota-Schulen“ (vgl. Wexler 1994) zeigt, wie sehr die Sprache der aktuellen Bildungspolitik mit der umstrukturierten, postfordistischen, postindustriellen Arbeitsorganisation übereinstimmt. Die nationale Vision der USA ist nicht mehr dem religiösen und politischen, sondern dem ökonomischen Sektor entlehnt. Die Produktivitätskrise und der globale ökonomische Wettbewerb verursachen den umstrukturierten Arbeitsplatz und indirekt die umstrukturierte Schule. Diese Entwicklung impliziert, dass die international standardisierte Rede über Bildung und nationale Entwicklung ursprünglich zwar eine westliche Ideologie war, aber nun nicht mehr vom Westen kontrolliert wird. Für den hier diskutierten Gegenstand ist zentral, dass gesellschaftliche Kohärenz nur noch in Begriffen des ökonomischen Diskurses herzustellen ist: in dieser Sprache können Nationen noch als Gemeinschaften imaginiert werden. Die Reform der Schule - dies ist vielleicht auch eine tragische Konsequenz der gescheiterten Gesellschaftsreform der 1960er - ist als nationales Anliegen nur im internationalen Wettbewerb legitimierbar. Bildungsleiter und Lehrer werden zu Teamleitern und Verkäufern von Unterricht als kundengelenkter und vom Arbeitsleben abgeleiteter Handelsware. Die Krise der Bildung wird als Designerproblem reformuliert. Die Bezeichnung „Toyota-Schule“ bezieht sich dabei auf ein Wirtschaftsunternehmen, das ironischerweise ausgerechnet dann in eine Krise geriet, als der Firmenname für Bildungsreformen adaptiert wurde.

\section{$3 \quad$ Schlussbemerkung}

Die multikulturelle pädagogische Auseinandersetzung um Differenz handelt weniger von der Passung zwischen Erziehungssystem und Arbeitsmarkt. Vielmehr geht es um die Analyse gesellschaftlicher Reproduktions- 
verhältnisse und schulischer Curricula als Spiegel des (nationalen) hegemonialen Diskurses. Die Multikulturalismusdebatte ist immer, gleich welche Prämissen einzelnen Varianten zugrunde liegen und welche Ziele sie verfolgen, mit gruppenbezogenen Identitäten, Repräsentationen, Forderungen usw. befasst. Weltsystemtheoretisch lassen sich die dargestellten Aspekte der pädagogischen Multikulturalismusdiskussion mit Überlegungen Balibars und Wallersteins kontextualisieren und erklären. Eine andere, hier ebenfalls thematisierte, weltsystemtheoretische Perspektive fokussiert den Zusammenhang von Individuum und Nation. Dieser Zugang verbindet Ökonomie und Schulsystem an der Schnittstelle Individuum und nutzt die Vorstellung eines ortlosen Weltkulturmodells. Er ist ebenfalls mit einem erziehungswissenschaftlich/pädagogisch relevanten Diskurs über Differenz verbunden: der liberal-humanistischen Position im Feminismus. Diese ist etabliert und findet sich häufig in einschlägigen wissenschaftlichen Monographien und Periodika. ${ }^{10}$

Zusammenfassen lässt sich, dass die Schwarze Bürgerrechtsbewegung, die die amerikanische Multikulturalimusdiskussion einleitete, den Pol „Kollektivität“ ansprach und die liberal feministische Diskussion den Pol "Individualität“. Beide Pole sind Teil des nationalstaatlichen Konstrukts, in dem - das zeigen die beiden weltsystemtheoretischen Zugänge - das Erziehungssystem immer eine herausragende Rolle spielt, gleich, ob die Integrations- und Sozialisationsfunktion oder die soziale und ökonomische Allokationsfunktion angesprochen sind. Die ungleich größere Rolle des Individualismus in feministischen Theorien verdeutlicht, dass im US-amerikanischen Kontext „Gender” und „Race” mit unterschiedlichen politischen und theoretischen Strategien verbunden sind. Im Zusammenhang mit einer Diskussion der Kontroverse um den ehemaligen Footballspieler O. J. Simpson brachte Michael Eric Dyson den Zusammenhang von individuel-

10 Da jedes Subjekt Ursprung der Selbsterkenntnis und des Wissens über die Welt ist, impliziert der liberal-humanistische Diskurs, die bestehenden Gesellschaftsverhältnisse seien letztlich gerechtfertigt. Diese Variante war auch in der Pädagogik bedeutend: Seit den 1970er Jahren sind Fragen der Chancengleichheit und des Bildungszugangs von Mädchen und Frauen Thema der Bildungsforschung und der Bildungspolitik; d. h., damit fand feministische Forschung am ehesten Anschluss an die Disziplin - u. a., weil dieser Diskurs auch anschlussfähig an das beschriebene Weltkulturmodell mit seiner Akzentuierung des modernen Individualismus ist. Die weltweit verbreitete, von staatlicher Organisation, wirtschaftlichem Entwicklungsstand und religiösen Präferenzen unabhängige Forderung nach Bildungsinklusion von Mädchen und Frauen unterstreicht, dass dies den Kern des modernen aus dem Weltkulturzusammenhang abgeleiteten nationalen Selbstverständnisses betrifft. Eine erste Sichtung angloamerikanischer Beiträge zur feministischen Pädagogik bestätigt den Konnex von Nationalstaat und Weltkultursemantik, staatlicher Bildung und Akzeptanz feministischer Pädagogik. Damit ist auch die umgekehrte Beobachtung vereinbar. Die „Differenzdiskussion“" in der Vergleichenden Erziehungswissenschaft zeigt, dass die Bereitschaft zunimmt, poststrukturalistisch inspirierte pädagogische Beiträge zu akzeptieren, je pluraler die Analyseeinheiten definiert und je weniger sie nur auf den Nationalstaat bezogen werden. 


\section{ler Leistung und Gruppenrepräsentation so auf den Punkt:}

„For many whites, excellence made blacks exceptions to, not examples of, their race. Ironically, to be thought of as an exception to the race still denied a pure consideration of individual merit. As long as race colored the yardstick, a real measurement of individual achievement was impossible. It is a bitter paradox that the evaluation of individual achievement that blacks yearned for was subordinated to a consideration of any achievement's impact on, and relation to, the race. Blacks were routinely denied the recognition of individual talent that is supposed to define the American creed“ (Dyson 1996, S. 18).

Die gruppenbezogenen Strategien der 1960er, die auf bestimmte gesellschaftliche Probleme reagierten, trugen zur (Re-)Produktion der beklagten Verhältnisse bei. Fraglich ist, wie auf aktuelle Probleme des sozialen Ausschlusses - in die weitere „Differenzen“ eingelagert sind - unter den veränderten Vorzeichen nationaler Semantik angemessen reagiert werden kann.

\section{Literatur}

Ackers, Sandra 1987: Feminist Theory and the Study of Gender and Education. In: International Review of Education, vol. 33, No.4 (1987), S. 419-435

Apple, Michael W. 1982: Power and Ideology. London: Routledge and Kegan Paul

Apple, Michael W. 1986: Teachers and Texts. A Political Economy of Class and Gender Relations in Education. New York: Routledge and Kegan Paul

Apple, Michael W. 1993: Official Knowledge: Democratic Education in a Conservative Age. London: Routledge

Apple, Michael W./Christian-Smith Linda 1991: The Politics of the Textbook, London, New York: Routledge 1991

Apple, Michael W./Weis, Lois (Hg.) 1983: Ideology and Practice in Schooling. Philadelphia: Temple University Press

Ashcroft, Bill/Griffiths, Gareth/Tiffin, Helen (Hg.) 1995: The Postcolonial Studies Reader. New York, London: Routledge

Auletta, Ken 1982: The Underclass. New York: Random House (Rev. and updated edition by Penguin USA, 2000)

Balibar, Etienne 1998: Die Nation-Form: Geschichte und Ideologie. In: Balibar/Wallerstein 1998, S. 107-129

Balibar, Etienne/Wallerstein, Immanuel 1998: Rasse, Klasse, Nation: Ambivalente Identitäten. 2. Auflage. Hamburg: Argument

Becker, Howard S. 1963: Outsiders. Studies in the Sociology of Deviance. London: Free Press

Bourdieu, Pierre 1989: How Schools Help Reproduce the Social Order. In: Current Contents/Social and Behavioral Science $21(8,1989)$, S. $16 \mathrm{ff}$.

Bourdieu, Pierre/Passeron, Jean-Claude 1971: Die Illusion der Chancengleichheit. Stuttgart: Klett

Bourgois, Philippe 1996: In Search of Respect. Selling Crack in El Barrio. Cambridge, Mass.: Harvard University Press

Bloom, Allan 1988: The Closing of the American Mind. New York: Touchstone Books

Boli, John/Ramirez, Francisco O./Meyer, John W. 1985: Explaining the Origins and Expansion of Mass Education. Comparative Education Review, vol. 29, no 2 (1985): S. $145-170$ 
Bowles, Samuel/Gintis, Herbert 1976: Schooling in Capitalist America. Educational Reform and the Contradictions of Modern Life. New York: Basic Books

Bradley, Karen/Ramirez, Francisco O. 1996: World Polity Promotion of Gender Parity: Women's Share of Higher Education, 1965-85. In: Research in Sociology of Education and Socialization 11, 1996, S. 63-91

Bromley, Roger/Götllich, Udo/Winter, Carsten (Hg.) 1999: Cultural Studies. Grundlagentexte zur Einführung. Lüneburg: zu Klampen

Bronfen, Elisabeth/Marius, Benjamin/Steffen, Therese (Hg.) 1997: Hybride Kulturen. Beiträge zur anglo-amerikanischen Multikulturalismusdebatte. Tübingen: Stauffenburg

Carnoy, Martin 1984: The State and Political Theory. Princeton: Princeton University Press

Carnoy, Martin/Levin, Henry M. 1985: Schooling and Work in the Democratic State. Stanford: Stanford University Press

Connors, Lyndsay/Mc Morrow, James 1988: National Policy Development: the Significance of the National Policy for the Education of Girls. In: Unicorn, Vol. 14, No. 4 (1988), S. 256-265.

D’Souza, Dinesh 1992: Illiberal Education. Political Correctness and the College Experience. John M. Ashbrook Center for Public Affairs Ashland University

Dyson, Michael Eric 1996: Race Rules. Navigating the Color Line. Reading, Mass. etc.: Addison-Wesley

Engelmann, Jan (Hg.) 1999: Die kleinen Unterschiede. Der Cultural Studies Reader. Frankfurt/M.: Campus

Fiala, Robert/ Gordon-Lanford, Audri 1987: "Educational Ideology and the World Educational Revolution, 1950-1970. Comparative Education Review 31 (3), S. 315-332

Fine, Michelle 1993: [Ap]parent Involvement. Reflections on Parents, Power, and Urban Public Schools. Teachers College Record, 94 (4, 1993): S. 682-710

Giroux, Henry 1983: Theory and Resistance in Education. South Hadley, Mass.: Bergin and Garvey

Giroux, Henry 1992: Border Crossings: Cultural Workers and the Politics of Education. New York, London: Routledge

Giroux, Henry/McLaren, Peter (Hg.) 1989: Critical Pedagogy: The State and Cultural Struggle. New York: State University of New York Press

Gitlin, Todd 1995: The Twilight of Common Dreams: Why America is Wracked by Culture Wars. New York: Metropolitan Books

Goffman, Erwin 1961: Asylums. Essays on the Social Situation of Mental Patients and other Inmates. Garden City, New York: Anchor Books

Grossberg, Lawrence/Nelson, Cary/Teichler, Paula (Hg.) 1992: Cultural Studies. New York: Routledge

Hirsch, E. D. 1988: Cultural Literacy. What Every American Needs to Know. New York: Vintage Books

Hörning, Karl/Winter, Rainer (Hg.) 1999: Widerspenstige Kulturen. Cultural Studies als Herausforderung. Frankfurt/M.: Suhrkamp

Jencks, Christopher/Peterson, Paul E. (Hg.) 1991: The Urban Underclass. Washington, D.C.: The Brookings Institute

Katz, Michael B. 1971: Class, Bureaucracy, and Schools: The Illusion of Educational Change in America. New York: Praeger

Katz, Michael B. (Hg.) 1993: The Underclass Debate. Views from History. Princeton, New Jersey: Princeton University Press 
Katz, Michael B. 1995: Improving Poor People. The Welfare State, the ,Underclass', and Urban Schools as History. Princeton: Princeton University Press

Kelly, Gail P./Younus, Lulat 1980: Women and Schooling in the Third World. In: Comparative Education Review, vol. 24 (June 1980), S. 224-263

Lewis, Dan A./Nakagawa, Kathryn 1995: Race and Educational Reform. A Study of School Decentralization. Albany: State University of New York Press

Loomba, Ania 1998: Colonialism/Postcolonialism. London, New York: Routledge

Massey, Douglas/Denton, Nancy 1994: American Apartheid. Segregation and the Making of the Underclass. Cambridge Mass.: Harvard University Press

Meier, Deborah 1995: The Power of Their Ideas. Lessons from a Small School in Harlem. Boston: Beacon Press

Meyer, John W. 1986: Myth of Personality and Socialization. In: Heller, Thomas C./ Sosna, Morton/Wellbery, David E. (Hg.) 1986: Reconstructing Individualism: Autonomy, Individuality, and the Self in Western Thought. Stanford, Cal.: Stanford University Press, S. 208-221

Meyer, John W./Boli, John/Thomas, George M./Ramirez, Francisco O. 1997: World Society and the Nation State. In: American Journal of Sociology, vol. 103, No.1 (July 1997), S. 144-181

Meyer, John W./Hannan, Michael T. 1979: National Development and the World System. Educational, Economic and Political Change, 1950 - 1970. Chicago: University of Chicago Press

Meyer, John W./Kamens, David/Benavot, Aaron mit Yun-Kyung und Suk-Ving Wong 1992: School Knowledge for the Masses: World Models and National Primary Curricular Categories for the Twentieth Century. London: Falmer Press

Morley, David/Chen, Kuan-Hsing 1996: Stuart Hall. Critical Dialogues in Cultural Studies. London, New York: Routledge

Moynihan, Daniel Patrick 1965: The Negro Family: The Case for National Action. Washington, D.C.: Department of Labor, Office of Policy Planning and Research

Omi, Michael/Winant, Howard 1994: Racial Formation in the United States. From the 1960s to the 1990s. London, New York: Routledge

Perry, Theresa/Delpit, Lisa 1998: The Real Ebonics Debate: Power, Language, and the Education of African-American Children. Boston: Beacon Press

Ramirez, Francisco O./Rubinson, Richard 1979: Creating Members: The Political Incorporation and Expansion of Public Education. In: Meyer/Hannan 1979, S. 72-82

Ramirez, Francisco O./Weiss, Jane 1979: The Political Incorporation of Women. In: Meyer/Hannan 1979, S. 238-249

Reed, Ishmael (Hg.) 1996: MultiAmerica: Essays on Cultural Wars and Cultural Peace. New York: Viking

Siegel, Fred 1997: The Future Once Happened Here. New York, D.C., L.A., and the Fate of America's Big Cities. New York, etc.: The Free Press

Surber, Jere Paul 1998: Culture and Critique. An Introduction to the Critical Discourses of Cultural Studies. Boulder, Colorado: Westview Press

Tyack, David B. 1974: The One Best System: A History of American Urban Education. Cambridge, Mass.: Harvard University Press

Wacquant, Loic J.D. 1994: Urban Outcasts. Color, Class, and Place in Two Advanced Societies. Ann Arbor: UMI Dissertation Information Service

Wallerstein, Immanuel 1998: Die Konstruktion von Völkern: Rassismus, Nationalismus, Ethnizität. In: Balibar/Wallerstein 1998, S. 87-106

Weibel, Peter/Zizek, Slavoj (Hg.) 1997: Inklusion: Exklusion. Probleme des Postkolonialismus und der globalen Migration. Wien: Passagen 
Wexler, Philip 1994: Die Toyota Schule. Ökonomisierung von Bildung und postmodernes Selbst. In: Sünker, Heinz/Timmermann, Dieter/Kolbe, Fritz-Ulrich (Hg.) 1994: Bildung, Gesellschaft, soziale Ungleichheit. Internationale Beiträge zur Bildungssoziologie und Bildungstheorie. Frankfurt/M.: Suhrkamp, S. 35-57

Wilson, William Julius 1987: The Truly Disadvantaged. The Inner City, the Underclass, and Public Policy. Chicago: The University of Chicago Press

Wilson, William Julius 1997: When Work Disappears. The World of the New Urban Poor. New York: Alfred A. Knopf

Žižek, Slavoj (Hg.) 1994: Mapping Ideology. London, New York: Verso 